\title{
EXISTENCE OF PERIODIC SOLUTIONS OF SECOND ORDER DIFFERENTIAL EQUATIONS WITH DELAY
}

\author{
GERHARD METZEN
}

(Communicated by Kenneth R. Meyer)

\begin{abstract}
We prove the existence of $2 \pi$-periodic solutions of second order ordinary differential equations with delay. In particular we study the effect the delay has with respect to the solvability of such problems. Resonance and nonresonance problems are considered.
\end{abstract}

1. Introduction. The purpose of this paper is to show how one can make effective use of the delay in proving the existence of periodic solutions. We are interested in certain Duffing type differential equations. In particular we consider

$$
\begin{aligned}
-x^{\prime \prime}(t)+c x^{\prime}(t) & =g(t, x(t-\tau))+f(t), \quad t \in(0,2 \pi), \\
x(0)-x(2 \pi) & =0=x^{\prime}(0)-x^{\prime}(2 \pi)
\end{aligned}
$$

where $g:(0,2 \pi) \times \mathbf{R} \rightarrow \mathbf{R}$ is a Carathéodory function with at most linear growth in $x$ and $f \in L^{1}(0,2 \pi)$. We also assume that $c \in \mathbf{R}, \tau \in[0,2 \pi)$ and $x \in W^{2,1}(0,2 \pi)$.

Problems such as (1) were discussed by de Pascale and Iannacci in [3] and Iannacci and Nkashama in [5 and 6]. See also Layton [9]. In these papers the authors applied techniques which were used to study problem (1) with $\tau=0$, that is, without delay. Conditions were formulated with respect to the spectrum of the problem

$$
\begin{gathered}
-x^{\prime \prime}(t)=\lambda x(t), \quad t \in(0,2 \pi), \\
x(0)-x(2 \pi)=0=x^{\prime}(0)-x^{\prime}(2 \pi) .
\end{gathered}
$$

In particular, in [6] it is shown that

$$
\begin{gathered}
-x^{\prime \prime}(t)=g(t, x(t-\tau))+f(t), \quad t \in(0,2 \pi), \\
x(0)-x(2 \pi)=0=x^{\prime}(0)-x^{\prime}(2 \pi)
\end{gathered}
$$

has a solution $x \in W^{2,1}(0,2 \pi)$ for every $f \in L^{1}(0,2 \pi)$ under the assumption that

$$
m^{2} \leq \alpha(t) \leq \liminf _{|s| \rightarrow \infty} g(t, s) / s \leq \limsup _{|s| \rightarrow \infty} g(t, x) / s \leq \beta(t) \leq(m+1)^{2},
$$

for some $m \in \mathbf{Z}$ and $\alpha, \beta \in L^{\infty}(0,2 \pi)$ with $m^{2}<\alpha(t)$ on a set of positive measure and likewise $\beta(t)<(m+1)^{2}$ on a set of positive measure.

In this paper we will extend the above result by studying the effect the delay $\tau$ has on the spectrum. In fact we also allow a damping term and so we study the

Received by the editors February 5, 1987 and, in revised form, April 14, 1987.

1980 Mathematics Subject Classification (1985 Revision). Primary 34B15, 34K10.

Key words and phrases. Delay, normal operator, periodic solution, resonance, nonresonance. 
spectrum of the problem:

$$
\begin{gathered}
-x^{\prime \prime}(t)+c x^{\prime}(t)=\lambda x(t-\tau), \quad t \in(0,2 \pi), \\
x(0)-x(2 \pi)=0=x^{\prime}(0)-x(2 \pi) .
\end{gathered}
$$

This is done in $\S 3$. In $\S 4$ we will study the nonresonance case and finally in $\S 5$ we allow resonance to occur.

2. Preliminaries. Throughout this paper we assume that all functions which are defined on either $(0,2 \pi)$ or $[0,2 \pi]$, are extended $2 \pi$-periodically over $\mathbf{R}$; i.e. $x(t)=x(t+2 \pi)$. For $\tau \in \mathbf{R}$ we define $T_{\tau}: L^{1}(0,2 \pi) \rightarrow L^{1}(0,2 \pi)$ by $\left(T_{\tau} x\right)(t):=$ $x(t-\tau)$. Let $\|\cdot\|_{p},\|\cdot\|_{m, n}$ and $|\cdot|_{k}$ denote the usual norms in the function spaces $L^{p}(0,2 \pi), W^{m, n}(0,2 \pi)$ and $C^{k}([0,2 \pi])$, respectively. Also let $\langle\cdot, \cdot\rangle$ denote the inner product in $L^{2}(0,2 \pi)$.

For $i \in\{1,2\}$ we define operators $L_{i}: \operatorname{dom}\left(L_{i}\right) \rightarrow L^{i}(0,2 \pi)$ by $\left(L_{i} x\right)(t):=$ $-x^{\prime \prime}(t)+c x^{\prime}(t)$, where $\operatorname{dom}\left(L_{i}\right):=\left\{x \in W^{2, i}(0,2 \pi) \mid x(0)-x(2 \pi)=0=x^{\prime}(0)-\right.$ $\left.x^{\prime}(2 \pi)\right\}$.

The operator $T_{\tau}$ has the following properties:

(i) $T_{\tau} T_{-\tau}=I=T_{-\tau} T_{\tau}$,

(ii) $T_{\tau}$ is an isometry on $L^{p}(0,2 \pi)$,

(iii) $\left(T_{\tau}\right)^{*}=T_{-\tau}$ as an operator in $L^{2}(0,2 \pi)$,

(iv) $L_{i} T_{\tau}=T_{\tau} L_{i}$ on $\operatorname{dom}\left(L_{i}\right), i=1,2$.

Next, let $g:(0,2 \pi) \times \mathbf{R} \rightarrow \mathbf{R}$ be a Carathéodory function satisfying: $|g(t, s)| \leq$ $c_{1}|s|+c_{2}(t)$, for all $(t, s) \in(0,2 \pi) \times \mathbf{R}$, where $c_{1}$ is some constant and $c_{2}(t)$ is some function in $L^{1}(0,2 \pi)$. With this we can of course define the substitution operator $N: L^{1}(0,2 \pi) \rightarrow L^{1}(0,2 \pi)$ by $(N x)(t):=g(t, x(t))$. Hence $\left(N T_{\tau} x\right)(t)=$ $g(t, x(t-\tau))$. Note that in general $N T_{\tau} \neq T_{\tau} N$.

Since we are interested in the influence of the delay, we consider a nonzero delay. Moreover it is clear that any delay problem can be reduced to a problem with $\tau \in[0,2 \pi)$, because the period is $2 \pi$. So let us fix some $\tau \in(0,2 \pi)$ and observe that problem (1) is equivalent to the operator equation

$$
L_{1} x=N T_{\tau} x+f .
$$

Since $g(t, s)$ has at most linear growth in $s$, it is important to study the case when $g(t, s)=\lambda s$. In this case $N=\lambda I$ and $N T_{\tau}=\lambda T_{\tau}$. We therefore study the equation $T_{-\tau} L_{1} x=T_{-\tau} N T_{\tau} x+T_{-\tau} f$. Let $L x:=T_{-\tau} L_{1} x$, then (1) is equivalent to

$$
L x=T_{-\tau} N T_{\tau} x+T_{-\tau} f .
$$

Because we intend to calculate the norm and the spectrum of the linear operator we study the operator $K:=T_{-\tau} L_{2}$, where $K: \operatorname{dom}(K) \subseteq L^{2}(0,2 \pi) \rightarrow L^{2}(0,2 \pi)$ and $\operatorname{dom}(K):=\operatorname{dom}\left(L_{2}\right)$. It is easily seen that $K$ is a closed, densely-defined, unbounded operator in a Hilbert space.

3. Properties of the linear operator $K$. One easily observes that $K$ is symmetric if and only if $c=0$ and $\tau=0$. Since we are interested in the case $\tau \in(0,2 \pi), K$ will never be selfadjoint. But $K$ has the following nice property. 
LEMMA 1. $K$ is a normal operator.

Proof. Clearly $K$ is densely defined and $K^{*}=\left(T_{-\tau} L_{2}\right)^{*}=L_{2}^{*} T_{\tau}$, by property (iii). Now it is shown in [4] that $L_{2}$ is normal. Therefore it follows that

$$
\begin{aligned}
\|K x\|_{2} & =\left\|T_{-\tau} L_{2} x\right\|_{2}=\left\|L_{2} x\right\|_{2}=\left\|L_{2}^{*} x\right\|_{2} \\
& =\left\|T_{\tau} L_{2}^{*} x\right\|_{2}=\left\|L_{2}^{*} T_{\tau} x\right\|_{2}=\left\|K^{*} x\right\|_{2}
\end{aligned}
$$

We have used (ii) and also the fact that $T_{\tau}$ commutes with $L_{2}^{*}$. Also $\operatorname{dom}(K)=$ $\operatorname{dom}\left(K^{*}\right)$ and hence $K$ is normal (cf. [11]). Q.E.D.

Next let $K_{\mathbf{C}}$ denote the complexification of the operator $K$ and let $L_{\mathbf{C}}^{2}(0,2 \pi)$ be the space of complex-valued square-integrable functions on $(0,2 \pi)$. Then

$$
\operatorname{dom}\left(K_{\mathbf{C}}\right):=\left\{x \in L_{\mathbf{C}}^{2}(0,2 \pi) \mid \operatorname{Re}(x), \operatorname{Im}(x) \in \operatorname{dom}\left(L_{2}\right)\right\} .
$$

Using Fouries series one easily shows that $\lambda_{n}:=\left(n^{2}+\operatorname{cin}\right) e^{i n \tau}, n \in \mathbf{Z}$, are eigenvalues of the operator $K_{\mathbf{C}}$ with corresponding eigenfunctions $\phi_{n}(t):=e^{i n t}$. We in fact have: Z\}.

LEMMA 2. $\sigma\left(K_{\mathbf{C}}\right)=\left\{\left(n^{2}+\operatorname{cin}\right) e^{i n \tau} \mid n \in \mathbf{Z}\right\}$ and $\sigma\left(K_{\mathbf{C}}^{*}\right)=\left\{\left(n^{2}-\operatorname{cin}\right) e^{i n \tau} \mid n \in\right.$

Proof. We already know that $\left.\left\{n^{2}+\operatorname{cin}\right) e^{i n \tau} \mid n \in \mathbf{Z}\right\} \subseteq \sigma\left(K_{\mathbf{C}}\right)$. Now let $\lambda \in \mathbf{C} \backslash\left\{\left(n^{2}+\operatorname{cin}\right) e^{i n \tau} \mid n \in \mathbf{Z}\right\}$ and let $y \in L_{\mathbf{C}}^{2}(0,2 \pi)$ be given. Let $y=\sum_{n \in \mathbf{Z}} a_{n} e^{i n t}$ and set $c_{n}:=a_{n}\left(\left(n^{2}+\operatorname{cin}\right) e^{i n \tau}-\lambda\right)^{-1}$. Since $\sum_{n \in \mathbf{Z}}\left|a_{n}\right|^{2}<\infty$, it follows that also $\sum_{n \in \mathbf{Z}}\left|c_{n}\right|^{2}<\infty, \sum_{n \in \mathbf{Z}}\left|n c_{n}\right|^{2}<\infty$ and $\sum_{n \in \mathbf{Z}}\left|n^{2} c_{n}\right|^{2}<\infty$. Therefore $x(t):=\sum_{n \in \mathbf{Z}} c_{n} e^{i n t} \in W_{C}^{2,2}(0,2 \pi)$ and $x(0)-x(2 \pi)=0=x^{\prime}(0)-x^{\prime}(2 \pi)$. Hence $x \in \operatorname{dom}\left(K_{\mathbf{C}}\right)$ and $\left(K_{\mathbf{C}}-\lambda\right) x=y$. Thus $K_{C}-\lambda$ is surjective and since $K_{\mathbf{C}}-\lambda$ is normal it follows that $\lambda \in \rho\left(K_{\mathbf{C}}\right)$. Therefore $\sigma\left(K_{\mathbf{C}}\right)=\left\{\left(n^{2}+\operatorname{cin}\right) e^{i n \tau} \mid n \in \mathbf{Z}\right\}$. Similarly we show that $\sigma\left(K_{\mathbf{C}}^{*}\right)=\left\{\left(n^{2}-c i n\right) e^{i n \tau} \mid n \in \mathbf{Z}\right\}$. Q.E.D.

One easily observes that $(K-\lambda)^{-1}: L^{2}(0,2 \pi) \rightarrow\left(\operatorname{dom}(K),|\cdot|_{1}\right)$ is a compact operator for all $\lambda \in \rho(K)$. The fact that $K$ is normal also implies that $\left\|(K-\lambda)^{-1}\right\|=$ $\left[\operatorname{dist}\left(\lambda, \sigma\left(K_{\mathbf{C}}\right)\right)\right]^{-1}$, for all $\lambda \in \rho(K)$.

REMARKS. (1) Let us consider the case when $c=0$.

(a) If $\tau / \pi \notin \mathbf{Q}$, then all eigenvalues $\lambda_{n}=n^{2} e^{i n \tau}, n \in \mathbf{Z}$, are simple and $\lambda_{n} \notin \mathbf{R}$, if $n \neq 0$.

(b) If $\tau / \pi \in \mathbf{Q}$, then let $\tau / \pi=q / p$ in lowest terms. If $n \neq 0$ is a multiple of $p$, say $n=j p$, we have $\lambda_{n}=\lambda_{-n}=(-1)^{j q}(j q \pi / \tau)^{2}=(-1)^{j q} n^{2}$. The corresponding eigenfunctions are $\cos (j q \pi / \tau) t$ and $\sin (j q \pi / \tau) t$. For all other $n \in \mathbf{Z}$ the eigenvalue $\lambda_{n}$ is simple and $\lambda_{n} \in \mathbf{C} \backslash \mathbf{R}$.

(2) In general (i.e. $c \in \mathbf{R}$ ) we have that $\lambda_{n}=\left(n^{2}+c i n\right) e^{i n \tau}$ is a simple eigenvalue if and only if $\lambda_{n} \notin \mathbf{R}$. If $\left(n^{2}+\operatorname{cin}\right) e^{i n \tau} \in \mathbf{R}$, then $\lambda_{n}=\lambda_{-n}$ and the corresponding eigenfunctions are $\cos (n t)$ and $\sin (n t)$, for $n \in \mathbf{Z} \backslash\{0\}$. So all real nonzero eigenvalues have multiplicity two and all other eigenvalues are simple.

(3) If $\lambda_{n} \in \sigma\left(K_{\mathbf{C}}\right)$ is real, then also $\lambda_{n} \in \sigma\left(K_{\mathbf{C}}^{*}\right)$ and conversely. So we have $\sigma\left(K_{\mathbf{C}}\right) \cap \mathbf{R}=\sigma\left(K_{\mathbf{C}}^{*}\right) \cap \mathbf{R}$.

4. Nonresonance case. In this section we will show that if $g(t, s)$ satisfies condition (A1) below, then for every $f \in L^{1}(0,2 \pi)$ we can find a solution of problem (1), or equivalently of problem (6). Let us fix some $\omega \in \mathbf{R} \backslash \sigma\left(K_{\mathbf{C}}\right)$ and set $r:=$ $\operatorname{dist}\left(\omega, \sigma\left(K_{\mathbf{C}}\right)\right)$. We assume that the nonlinearity $g$ satisfies the following condition: 
(A1) $g:(0,2 \pi) \times \mathbf{R} \rightarrow \mathbf{R}$ is a Carathéodory function such that

(i) for every $R>0$, there exists some $f_{R} \in L^{1}(0,2 \pi)$ so that $|g(t, s)| \leq f_{R}(t)$, for all $t \in(0,2 \pi)$ and $|s| \leq R$.

(ii) there exist some functions $\alpha, \beta \in L^{\infty}(0,2 \pi)$ and a set of positive measure, $P \subseteq(0,2 \pi)$, such that:

$$
\omega-r \leq \alpha(t) \leq \liminf _{|s| \rightarrow \infty} g(t, s) / s \leq \limsup _{|s| \rightarrow \infty} g(t, s) / s \leq \beta(t) \leq \omega+r,
$$

uniformly in $t \in(0,2 \pi)$ and $\omega-r<\alpha(t) \leq \beta(t)<\omega+r$, for all $t \in P$.

LEMMA 3. Let $p \in L^{\infty}(0,2 \pi)$ satisfy $\alpha(t) \leq p(t) \leq \beta(t)$, for all $t \in(0,2 \pi)$. Then $L x=p x$ implies $x=0$.

ProOF. Now $p \in L^{\infty}(0,2 \pi)$ and $x \in W^{2,1}(0,2 \pi)$ implies that $p x \in L^{2}(0,2 \pi)$. Therefore, $L x=p x$ implies that in fact $x \in W^{2,2}(0,2 \pi)$ and $K x=p x$. Suppose that there exists some $x_{0} \neq 0$ with $K x_{0}=p x_{0}$. Clearly there are only finitely many $\lambda_{1}, \ldots, \lambda_{k} \in \sigma\left(K_{\mathbf{C}}\right)$ which lie on the circle $|\omega-z|=r$. Let

$$
V:=\bigoplus_{i=1}^{k} \operatorname{Ker}\left(K_{\mathbf{C}}-\lambda_{i}\right)
$$

then $\operatorname{dim}(V)<\infty$ since each eigenvalue has finite multiplicity. The nonzero functions in $V$ have finitely many zeroes in $[0,2 \pi]$ since they are analytic.

We have $\left\|\left(K_{\mathbf{C}}-\omega\right) x\right\|_{2} \geq r\|x\|_{2}$, for all $x \in \operatorname{dom}\left(K_{\mathbf{C}}\right)$, and equality holds if and only if $x \in V$. On the other hand we also have $\|p x-\omega x\|_{2} \leq r\|x\|_{2}$, for all $x \in L_{\mathbf{C}}^{2}(0,2 \pi)$. Therefore $x_{0}$ satisfies $\left\|(K-\omega) x_{0}\right\|_{2}=r\left\|x_{0}\right\|_{2}$ and so $x_{0} \in V$. Since $|p(t)-\omega|<r$ on a set of positive measure, it follows that $\left\|(p-\omega) x_{0}\right\|_{2}<r\left\|x_{0}\right\|_{2}$. This contradiction proves the lemma. Q.E.D.

LEMMA 4. There exists some $\varepsilon>0$ so that $p \in L^{\infty}(0,2 \pi)$ with $\alpha(t)-\varepsilon \leq$ $p(t) \leq \beta(t)+\varepsilon$, for all $t \in(0,2 \pi)$, and $L x=p x$ implies $x=0$.

PROOF. If the lemma were false, then there exist sequences $\left\{p_{n}\right\} \subseteq L^{\infty}(0,2 \pi)$ and $\left\{x_{n}\right\} \subseteq \operatorname{dom}(L) \backslash\{0\}$ with $\alpha(t)-1 / n \leq p_{n}(t) \leq \beta(t)+1 / n$, for all $t \in(0,2 \pi)$, and $L x_{n}=p_{n} x_{n}$. As in the proof of Lemma 3 we may replace the operator $L$ by $K$. We also may assume that $\left\|x_{n}\right\|_{2}=1$ and that there exists some $p \in L^{\infty}(0,2 \pi)$ and $v \in L^{2}(0,2 \pi)$ so that $p_{n}-p$ and $(K-\omega) x_{n} \rightarrow v$ as $n \rightarrow \infty$. Set $x:=(K-\omega) v$, then $x_{n}-x$ in $L^{2}(0,2 \pi)$ as $n \rightarrow \infty$. Therefore $p_{n} x_{n} \rightarrow p x$. Now $p_{n} \rightarrow p$ and $\alpha(t)-1 / n \leq p_{n}(t) \leq \beta(t)+1 / n$ implies that $\alpha(t) \leq p(t) \leq \beta(t)$. We get that $K x=p x$ with $x \neq 0$. By Lemma 3 this is a contradiction. Q.E.D.

THEOREM 1. Let $g$ satisfy (A1) and let $f \in L^{1}(0,2 \pi)$, then equation (1) has a solution $x \in W^{2,1}(0,2 \pi)$.

ProOF. We let $\varepsilon>0$ be as in Lemma 4 and choose $R \in \mathbf{R}_{+}$so that $|s|>R$ implies $\alpha(t)-\varepsilon \leq g(t, s) / s \leq \beta(t)+\varepsilon$, for all $t \in(0,2 \pi)$. Define functions $g_{1}$, $h:(0,2 \pi) \times \mathbf{R} \rightarrow \mathbf{R}$ by

$$
g_{1}(t, s):= \begin{cases}g(t, s) s R^{-2}, & \text { if }|s| \leq R, \\ g(t, s) s^{-1}, & \text { if }|s|>R,\end{cases}
$$

and $h(t, s):=\max \left\{\alpha(t)-\varepsilon, \min \left\{\beta(t)+\varepsilon, g_{1}(t, s)\right\}\right\}$. Then $|g(t, s)-h(t, s) s| \leq \eta(t)$, $t \in(0,2 \pi)$, for some $\eta \in L^{1}(0,2 \pi)$. Let $N, H: L^{1}(0,2 \pi) \rightarrow L^{1}(0,2 \pi)$ denote 
the substitution operators corresponding to $g(t, s)$ and $h(t, s) s$, respectively. Then $\|N x-H x\|_{1} \leq c$, for all $x \in L^{1}(0,2 \pi)$. With $M:=L-\omega$ we see that equation (6) is equivalent to

$$
x=M^{-1} T_{-\tau}(N-H) T_{\tau} x+M^{-1} T_{-\tau}(H-\omega) T_{\tau} x+M^{-1} T_{-\tau} f .
$$

We now consider the homotopy

$$
x=t M^{-1} T_{-\tau}(N-H) T_{\tau} x+t M^{-1} T_{-\tau}(H-\omega) T_{\tau} x+t M^{-1} T_{-\tau} f,
$$

where $t \in[0,1]$, and establish an a priori bound for all possible solutions with respect to the $C^{1}$-norm. If an apriori bound did not exist, then we could find sequences $\left\{x_{n}\right\} \subseteq \operatorname{dom}(L)$ and $\left\{t_{n}\right\} \subseteq[0,1]$ such that $\left|x_{n}\right|_{1} \rightarrow \infty$ as $n \rightarrow \infty$ and equation (8) would be satisfied with $x=x_{n}$ and $t=t_{n}$. Let $v_{n}:=x_{n}\left|x_{n}\right|_{1}^{-1}$ and

$$
r_{n}:=\left|x_{n}\right|_{1}^{-1} t_{n} M^{-1} T_{-\tau}(N-H) T_{\tau} x_{n}+\left|x_{n}\right|_{1}^{-1} t_{n} M^{-1} T_{-\tau} f .
$$

Then $\lim _{n \rightarrow \infty} r_{n}=0$ in $C^{1}([0,2 \pi])$ and $v_{n}=r_{n}+t_{n}\left|x_{n}\right|_{1}^{-1} M^{-1} T_{-\tau}(H-\omega) T_{\tau} x_{n}$. Since $\left\{v_{n}\right\}$ is bounded in $C^{1}([0,2 \pi])$, we may, without loss of generality, assume that $v_{n} \rightarrow v$ in $C([0,2 \pi])$, for some $v \in C([0,2 \pi])$. Set $y_{n}:=T_{\tau} x_{n}$ and $p_{n}(t):=$ $h\left(t, y_{n}(t)\right)$ and observe that

$$
t_{n}\left|x_{n}\right|_{1}^{-1} M^{-1} T_{-\tau}(H-\omega) T_{\tau} x_{n}=t_{n} M^{-1} T_{-\tau}\left(p_{n}-\omega\right) T_{\tau} v_{n}
$$

Again, we may assume that $t_{n} \rightarrow t^{*} \in[0,1]$ and that $p_{n}-p$ in $L^{2}(0,2 \pi)$, for some $p \in L^{2}(0,2 \pi)$. Note that $\alpha(t)-\varepsilon \leq p(t) \leq \beta(t)+\varepsilon$, for all $t \in(0,2 \pi)$. We obtain that $v=t^{*} M^{-1} T_{-\tau}(p-\omega) T_{\tau} v$. With $x:=T_{\tau} v$ we get

$$
M x=t^{*}(p-\omega) x=\left[t^{*} p+\left(1-t^{*}\right) \omega\right] x-\omega x .
$$

Therefore $L x=\left[t^{*} p+\left(1-t^{*}\right) \omega\right] x$ and of course

$$
\alpha(t)-\varepsilon \leq t^{*} p(t)+\left(1-t^{*}\right) \omega \leq \beta(t)+\varepsilon,
$$

for all $t \in(0,2 \pi)$. This contradicts Lemma 4 . Therefore all solutions of equation (8) are bounded and so by the compactness of $M^{-1}$ we obtain a solution to equation (7). Q.E.D.

REMARKS. (1) Theorem 1 extends the main result of [6] because when $c=0$ and $\tau \in(0,2 \pi)$ then it is impossible to have $\left\{\lambda_{n}, \lambda_{n+1}\right\} \subseteq \mathbf{R}_{+}$or $\left\{\lambda_{n}, \lambda_{n+1}\right\} \subseteq \mathbf{R}_{-}$. For example if $c=0$ and $\tau=\pi$, then we can take $\omega-r=(2 m)^{2}$ and $\omega+r=(2 m+2)^{2}$, or $\omega-r=-(2 m+3)^{2}$ and $\omega+r=-(2 m+1)^{2}, m \geq 0$, or $\omega-r=-1$ and $\omega+r=0$, in Theorem 1. Therefore the assumption

$$
m^{2} \leq \alpha(t) \leq \liminf _{|s| \rightarrow \infty} g(t, s) / s \leq \limsup _{|s| \rightarrow \infty} g(t, s) / s \leq \beta(t) \leq(m+1)^{2}
$$

can be replaced by

$$
(2 m)^{2} \leq \alpha(t) \leq \liminf _{|s| \rightarrow \infty} g(t, s) / s \leq \limsup _{|s| \rightarrow \infty} g(t, s) / s \leq \beta(t) \leq(2 m+2)^{2}
$$

or by

$$
-(2 m+3)^{2} \leq \alpha(t) \leq \liminf _{|s| \rightarrow \infty} g(t, s) / s \leq \limsup _{|s| \rightarrow \infty} g(t, s) / s \leq \beta(t) \leq-(2 m+1)^{2}
$$

or by

$$
-1 \leq \alpha(t) \leq \liminf _{|s| \rightarrow \infty} g(t, s) / s \leq \limsup _{|s| \rightarrow \infty} g(t, s) / s \leq \beta(t) \leq 0
$$


(2) Theorem 1 partially extends certain results of [5] as well.

(3) Our ideas can also be applied to systems with delay as considered by Layton in [9]. It is also possible to allow different delays to appear in the differential equation. Details will appear elsewhere.

5. Resonance case. Resonance here means that we allow

$$
\omega-r=\liminf _{|s| \rightarrow \infty} g(t, s) / s
$$

to occur. In this section we consider resonance at $\omega-r=0$ only. Results about resonance at other real eigenvalues will appear elsewhere in a more general and abstract setting. We will prove two theorems in this section. In one we assume a Landesman-Lazer condition holds and in the other we do not.

We make the following assumptions about the nonlinearity $g$ :

(A2) There exist $r, \omega \in \mathbf{R}_{+}$so that either

or:

(i) $\operatorname{cl}\left(B_{r}(\omega)\right) \cap \sigma\left(K_{\mathbf{C}}\right)=\{0, \omega+r\}$

(ii) $\operatorname{cl}\left(B_{r}(\omega)\right) \cap \sigma\left(K_{\mathbf{C}}\right)=\{0\}$.

Here $B_{r}(\omega):=\{z \in \mathbf{C}|| \omega-z \mid<r\}$.

(A3) $g:(0,2 \pi) \times \mathbf{R} \rightarrow \mathbf{R}$ is a Carathéodory function such that

(i) for every $R>0$, there exists some $f_{R} \in L^{1}(0,2 \pi)$ so that $|g(t, s)| \leq f_{R}(t)$, for all $t \in(0,2 \pi)$ and $|s| \leq R$.

(ii) there exists some function $\beta \in L^{\infty}(0,2 \pi)$ and a set of positive measure, $P \subseteq(0,2 \pi)$, such that

$$
0 \leq \limsup _{|s| \rightarrow \infty} g(t, s) / s \leq \beta(t) \leq \omega+r,
$$

for all $(t, s) \in(0,2 \pi) \times \mathbf{R}$ and $\beta(t)<\omega+r$, for all $t \in P$.

(iii) there exists some $M>0$ and some $c \in L^{1}(0,2 \pi)$ so that $s(g(t, s)-c(t)) \geq 0$, for all $t \in(0,2 \pi)$ and $|s| \geq M$.

LEMMA 5. Let $r, \omega$, and $\beta$ be as in (A2)-(A3). Then $p \in L^{\infty}(0,2 \pi)$ with $0 \leq p(t) \leq \beta(t)$, for all $t \in(0,2 \pi)$, and $L x=p x$ implies that $x \in \operatorname{Ker}(L)$.

PrOOF. Since the case when (A2) (ii) holds is easier than when (A2) (i) holds, we will consider (A2) (i) only. Suppose that $x_{0} \in \operatorname{dom}(L)$ with $L x_{0}=p x_{0}$. As in the proof of Lemma 3 we can easily show that $x_{0} \in \operatorname{Ker}(L) \oplus \operatorname{Ker}(L-(\omega+r))$. Set $x_{0}=x_{1}+x_{2} \in \operatorname{Ker}(L) \oplus \operatorname{Ker}(L-(\omega+r))$. Then

$$
\begin{aligned}
& \left\langle L x_{0}, x_{1}\right\rangle=0=\left\langle p x_{1}, x_{1}\right\rangle+\left\langle p x_{2}, x_{1}\right\rangle \text { and } \\
& \left\langle L x_{0}, x_{2}\right\rangle=(\omega+r)\left\|x_{2}\right\|^{2}=\left\langle p x_{1}, x_{2}\right\rangle+\left\langle p x_{2}, x_{2}\right\rangle .
\end{aligned}
$$

Therefore $(\omega+r)\left\|x_{2}\right\|^{2}=\left\langle p x_{2}, x_{2}\right\rangle-\left\langle p x_{1}, x_{1}\right\rangle$. Since $0 \leq p(t)<\omega+r$, for all $t \in P$, it follows that $\left\|x_{2}\right\|=0$. Hence $\left\langle p x_{1}, x_{1}\right\rangle=0$ and so $x_{0}=x_{1} \in \operatorname{Ker}(L)$ and $p(t)=0$, for all $t \in(0,2 \pi)$. Q.E.D.

Using a proof similar to the proof of Lemma 4 we can show that the following lemma holds.

LEMMA 6. Let $r, \omega$, and $\beta$ be as in (A2)-(A3). Then there exists some $\varepsilon>0$ so that $p \in L^{\infty}(0,2 \pi)$ with $0 \leq p(t) \leq \beta(t)+\varepsilon$, for all $t \in(0,2 \pi)$, and $L x=p x$ implies $x \in \operatorname{Ker}(L)$. 
We now can prove the following

THEOREM 2. Let $g$ satisfy (A2) and (A3) and let $f \in L^{1}(0,2 \pi)$ satisfy

$$
\int_{0}^{2 \pi} g(t,-\infty) d t<-\int_{0}^{2 \pi} f(t) d t<\int_{0}^{2 \pi} g(t, \infty) d t
$$

where $g(t,-\infty):=\limsup _{s \rightarrow-\infty} g(t, s)$ and $g(t, \infty):=\liminf _{s \rightarrow \infty} g(t, s)$. Under these assumptions equation (1) has a solution $x \in W^{2,1}(0,2 \pi)$.

PROOF. Let $\varepsilon>0$ be as in Lemma 6. If $1 / n<\varepsilon, n \in \mathbf{N}$, then $L x-(1 / n) x=$ $T_{-\tau} N T_{\tau} x+T_{-\tau} f$ is a nonresonance problem and hence it has a solution $x_{n} \in$ $W^{2,1}(0,2 \pi)$. In case $\left\{\left|x_{n}\right|_{1}\right\}$ is bounded, one easily sees that we obtain a solution of (1). So let us assume that $\lim _{n \rightarrow \infty}\left|x_{n}\right|_{1}=\infty$ (by choosing a subsequence) and let $v_{n}:=x_{n}\left|x_{n}\right|_{1}^{-1}$. Without loss of generality we also may assume that $v_{n} \rightarrow v$ in $C([0,2 \pi])$, for some $v \in C([0,2 \pi])$. Define functions $g_{1}(t, s)$ and $h(t, s)$ as in the proof of Theorem 1 , with the necessary modifications, and let $H$ denote the substitution operator corresponding to $h$. With $M:=L-\omega$ we get

$x_{n}-(1 / n) M^{-1} x_{n}=M^{-1} T_{-\tau}(N-H) T_{\tau} x_{n}+M^{-1} T_{-\tau}(H-\omega) T_{\tau} x_{n}+M^{-1} T_{-\tau} f$.

Proceeding as in the proof of Theorem 1 we eventually obtain

$$
v=M^{-1} T_{-\tau}(p-\omega) T_{\tau} v \quad \text { and } \quad L x=p x,
$$

where $x:=T_{\tau} v$ and $p \in L^{\infty}(0,2 \pi)$ satisfies $0 \leq p(t) \leq \beta(t)+\varepsilon$, for all $t \in(0,2 \pi)$. Therefore $x \in \operatorname{Ker}(L)$ by Lemma 6 and since $x \neq 0$ we may assume that $x>0$.

Now

$$
\left\langle L x_{n}, x\right\rangle-(1 / n)\left\langle x_{n}, x\right\rangle=\left\langle T_{-\tau} N T_{\tau} x_{n}, x\right\rangle+\left\langle T_{-\tau} f, x\right\rangle
$$

and therefore

$$
\frac{-1}{n}\left\langle x_{n}, x\right\rangle=\int_{0}^{2 \pi} g\left(t+\tau, x_{n}(t)\right) x d t+\int_{0}^{2 \pi} f(t+\tau) x d t .
$$

Using assumption (A3)(iii) and $\lim _{n \rightarrow \infty}\left|x_{n}\right|_{1}=\infty$ we can apply Fatou's lemma and obtain

$$
\begin{aligned}
x \int_{0}^{2 \pi} g(t+\tau, \infty) & d t+x \int_{0}^{2 \pi} f(t+\tau) d t \\
\leq & \liminf _{n \rightarrow \infty}\left[\int_{0}^{2 \pi} g\left(t+\tau, x_{n}(t)\right) x d t+\int_{0}^{2 \pi} f(t+\tau) x d t\right] \\
& =\liminf _{n \rightarrow \infty}\left[-1 / n\left\langle x_{n}, x\right\rangle\right] \leq 0 .
\end{aligned}
$$

This contradicts condition (9). Therefore $\left\{\left|x_{n}\right|_{1}\right\}$ is bounded and we have a solution. The same conclusion is reached if we assume $x<0$. Q.E.D.

If the nonlinearity $g$ satisfies $g(t,-\infty)=g(t, \infty)$, then condition (9) cannot be satisfied by any $f \in L^{1}(0,2 \pi)$. A particularly simple condition in this case was given by De Figueiredo and $\mathrm{Ni}$ in [2]. This result was generalized from bounded to unbounded nonlinearities by Iannacci and Nkashama [7] and Ward [10].

Following the proof of Theorem 2 we can easily prove the next theorem. 
THEOREM 3. Assume that $g$ satisfies (A2) and (A3). Let $f \in L^{1}(0,2 \pi)$ satisfy

$$
-\int_{0}^{2 \pi} f(t) d t=\int_{0}^{2 \pi} c(t) d t .
$$

Then equation (1) has a solution $x \in W^{2,1}(0,2 \pi)$.

REMARKS. (1) Theorem 3 improves a result in [3, Theorem 3] if it is restricted to a Duffing equation such as (1). The authors consider a Lienard equation and assume $\beta(t) \leq 1$ in condition (A3) (ii).

(2) We can use the same counterexample as in [8] to show that Theorem 3 does not hold for eigenvalues $0 \neq \lambda_{n} \in \mathbf{R}$.

\section{REFERENCES}

1. D. G. de Figueiredo, Semilinear elliptic equations at resonance: Higher eigenvalues and unbounded nonlinearities, Advances in Differential Equations (Roberto Conti, ed.), Academic Press, New York, 1981, pp. 89-99.

2. D. G. de Figueiredo and W. M. Ni, Perturbations of second order linear elliptic problems by nonlinearities without Landesman-Lazer condition, Nonlinear Anal. TMA 3 (1979), 629-634.

3. E. de Pascale and R. Iannacci, Periodic solutions of generalized Lienard equations with delay, Equadiff 82, Würzburg, Lecture Notes in Math., vol. 1017, Springer-Verlag, Berlin and New York, 1983 pp. 148-156.

4. P. Habets and G. Metzen, Periodic solutions of Duffing equations J. Differential Equations (to appear).

5. R. Iannacci and M. N. Nkashama, Nonresonance conditions for periodic solutions of forced Lienard and Duffing equations with delay, Ann. Soc. Sci. Bruxelles 99 (1985), 29-43.

6. __ On periodic solutions of forced second order differential equations with deviating argument, Ordinary and Partial Differential Equations, Proc. (Dundee, U. K. 1984), Lecture Notes in Math., vol. 1151, Springer-Verlag, Berlin, 1985.

7. __ Nonlinear two point boundary value problems as resonance without Landesman-Lazer's condition (preprint).

8. _ Unbounded perturbations of forced second order ordinary differential equations at resonance J. Differential Equations 69 (1987), 289-309.

9. W. Layton, Periodic solutions of nonlinear delay equations, J. Math. Anal. Appl. 77 (1980), 198-204.

10. J. R. Ward, A note on the Dirichlet problem for some semilinear elliptic equations (preprint).

11. T. Weidman, Lineare Operatoren in Hilberträumen, Teubner, Stuttgart, 1976.

Department of Mathematical Sciences, Memphis State UniVersity, Memphis, TENNESSEE 38152 